\title{
Relativistic jets and non-thermal radiation from collapse of stars to black holes
}

\author{
V. Kryvdyk and A. Agapitov \\ Depart. of Astronomy, Kyiv National University, av. Glushkova 2/1, Kyiv 03022, Ukraine \\ email: kryvdyk@univ.kiev.ua
}

\begin{abstract}
The formation of the relativistic jets and a non-thermal emission from the collapsing magnetized stars with dipole magnetic fields and the heterogeneous particles distribution are investigated. These polar jets are formed when the stellar magnetosphere compress during collapse its magnetic field increases considerable. The electric field is produced in magnetosphere, which the charged particles will be accelerated. As follow from the calculation, the jets can be formed from collapsing stars already the explosion of supernova stars without shock waves. These jets will generate the non-thermal radiation. The radiation flux depends on the distance to the star, its magnetic field and the particle spectrum in the magnetosphere. This flux can be observed near Earth by means of modern telescopes in the form of the radiation pulse with duration equal to time collapse.
\end{abstract}

Keywords. Collapsing stars - particle acceleration - non-thermal emission - relativistic jets

\section{Introduction}

In our previous papers (Kryvdyk 1999, 2004, 2005) we investigated the particles acceleration and the non-thermal emission from the collapsing magnetized stars. It was showed that the collapsing stars can be powerful sources of the non-thermal radiation, which can be observed by means of modern telescopes. In this paper we consider the formation of the relativistic jets by collapse of stars with heterogeneous magnetospheres to black holes. These jets formed when the stellar magnetosphere compress during the collapse and its magnetic field increases considerably. A cyclic electric field is produced and the charged particles are accelerated in polar caps of collapsing stars.

\section{Magnetosphere of collapsing star}

We examine particles in magnetospheres of collapsing stars with dipolar magnetic fields. We consider three cases of initial heterogeneous distributions of the particles power-series distribution, the relativistic Maxwell one, and the Boltzmann distribution,

$$
\begin{gathered}
N_{P}^{i}(E, R)=\left(r_{*}\right)^{3} K_{P} E_{*}^{-\gamma} R_{*}^{-\beta_{P}} \\
N_{M}^{\prime}(E, R)=\left(r_{*}\right)^{3} K_{M} E_{*}^{2} R_{*}^{-\beta_{M}} \exp (-E / k T), \\
N_{B}^{\prime}(E, R)=\left(r_{*}\right)^{3} K_{B} R_{*}^{-\beta_{B}} \exp (-E / k T),
\end{gathered}
$$

Here, $E_{*}=E / E_{0} ; r_{*}=r_{0} / r ; \beta_{P}=a_{1}(\gamma-1) ; \beta_{M}=a_{1}\left(E / k T \ln E_{*}-3\right) ; \beta_{B}=$ $a_{1}\left(E / k T \ln E_{*}-1\right) ; a_{1}=\left(5 k_{1} / 3\right)\left(3 \cos ^{4} \theta+1.2 \cos ^{2} \theta-1\right)\left(1+3 \cos ^{2} \theta\right)^{-2} ; K_{C}, K_{M}, K_{B}$ are the spectral coefficients, $k$ is the Boltzmann constant, and $T$ is temperature.

Figure 1 shows the transformation of the stellar magnetosphere during collapse. We can see that the initial stellar magnetosphere transforms during the collapse. The polar jets are formed in the magnetosphere already at the initial stage of collapse. Particle 


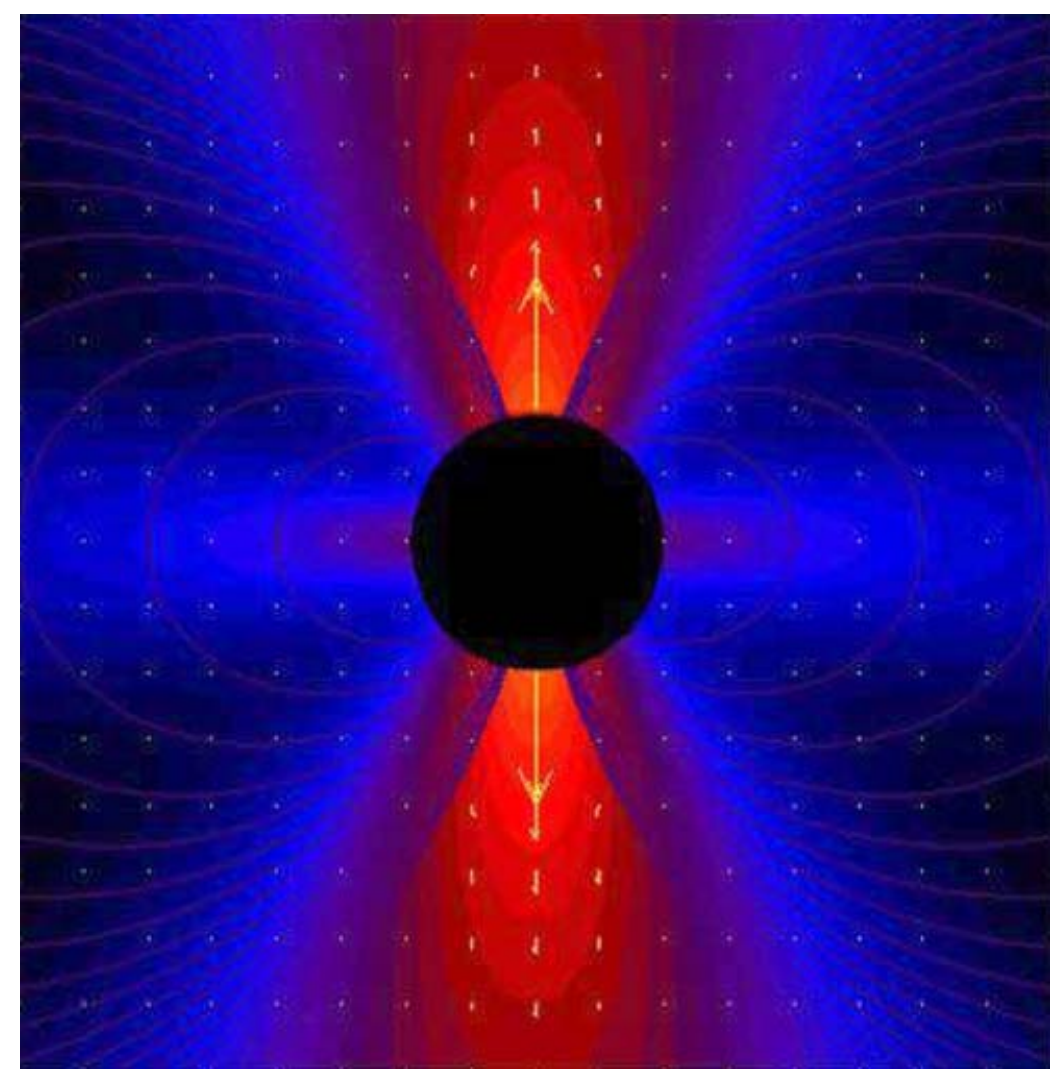

Figure 1. Relativistic jets emerging from a collapsing star. This simulation shows the structure of the stellar magnetosphere during collapse.

density and energy in polar jets grow during the process of collapse. Thus, the jets from collapsing stars can be formed already at the moment of the explosion of supernovae without shock waves.

\section{Conclusions}

As follows from our results, the charged particles are accelerated in the magnetosphere of collapsing stars to relativistic energy; the relativistic polar jets are formed. These jets emit the electromagnetic waves in all frequency bands. The radiation flux grows during collapse and reaches maximum at the final stage of collapse. This radiation can be observed as bursts in all frequency bands, from radio to gamma ray.

\section{References}

Kryvdyk, V. 1999, MNRAS, 309, 593

Kryvdyk, V. 2004, Adv. Sp. Res., 33, 484

Kryvdyk, V. \& Agapitov, A. 2005, ASP CS, 330, 415 